\title{
Comparison of transcutaneous and plasma bilirubin measurement
}

\author{
Maria das Graças da Cunha Leite, ${ }^{1}$ Valéria de Araújo Granato, ${ }^{2}$ \\ Fernando Perazzini Facchini, ${ }^{3}$ Sérgio Tadeu Martins Marba ${ }^{3}$
}

\begin{abstract}
Objectives: To compare transcutaneous bilirubin measurements made using Bilicheck equipment with assays of capillary plasma using the Unistat bilirubinometer (Leica).

Methods: Two hundred concomitant assays were performed (transcutaneous and in plasma), and the correlation and level of agreement between them was calculated. An assessment was also made of the influence of birth weight, skin color, gestational age, postnatal age and phototherapy.

Results: The linear correlation coefficient was 0.92 , and the mean difference between assays was $0.72( \pm 1.57)$ $\mathrm{mg} / \mathrm{dL}$, with a $95 \%$ confidence interval from -2.42 to +3.86 . The best of a series of ROC curves demonstrated that transcutaneous assays at $14 \mathrm{mg} / \mathrm{dL}$ offer the best sensitivity $(88.2 \%)$ and specificity $(97.8 \%)$, with a positive predictive value of $78.9 \%$, negative predictive value of 98.9 and are below the curve of 0.98 .
\end{abstract}

Conclusions: Assays performed using Bilicheck can be substituted for capillary plasma assays up to $14 \mathrm{mg} / \mathrm{dL}$. Above this level the device should only be used for screening for patients whose bilirubin should be assayed in blood.

J Pediatr (Rio J). 2007;83(3):283-286: Bilirubin, hyperbilirubinemia, jaundice, newborn, transcutaneous bilirubin measurement.

\section{Introduction}

Bilirubinemia assessment is the most important factor when managing neonatal jaundice. Major progress has been achieved in modernizing assay methods and increasing their reliability. The first apparatus for transcutaneous assessment was launched during the nineteen eighties, the Minolta ${ }^{\circledR}$ Bilirubinometer (Minolta Camera $\mathrm{Co}^{\circledR}$, Japan), and simply correlated yellow skin color with bilirubinemia. ${ }^{1}$ Later, the efforts of researchers resulted in the development of more precise equipment, attempting to achieve a real reduction in the number of blood-based bilirubin assays. ${ }^{2}$

Bilicheck $^{\circledR}$ (SpectRx Inc, Norcross, Georgia, USA) is an instrument that emits a shaft of light onto the skin and recaptures the light reflected. The light absorbed by the bilirubin is assessed after the portions affected by the quantity of collagen, melanin and hemoglobin have been duly accounted for. Initial studies with this type of equipment suggested that it would be possible to replace laboratory methods for measuring bilirubinemia by transcutaneous

1. Mestre, Faculdade de Ciências Médicas, Universidade Estadual de Campinas (UNICAMP), Campinas, SP, Brasil.

2. Médica residente, Unidade Neonatal, Centro de Atenção Integral à Saúde da Mulher, UNICAMP, Campinas, SP, Brasil.

3. Doutor. Professor assistente, Departamento de Pediatria, Faculdade de Ciências Médicas, UNICAMP, Campinas, SP, Brasil.

Manuscript received Aug 22 2006, accepted for publication Nov 142006.

Suggested citation: Leite MG, Granato VA, Facchini FP, Marba ST. Comparison of transcutaneous and plasma bilirubin measurement. J Pediatr (Rio J). 2007;83(3):283-286.

Financial support: Research supported by the Fundo de Apoio ao Ensino e à Pesquisa (FAEP), affiliated to the Universidade Estadual de Campinas (UNICAMP), Campinas, SP, Brazil.

doi 10.2223/JPED.1619 
measurement. ${ }^{3,4}$ More recent studies then cast doubt on the accuracy needed for this replacement, ${ }^{5,6}$ suggesting that Bilicheck (BC) should merely be used to screen hyperbilirubinemia.

The objective of the current study is to compare transcutaneous and plasma bilirubin measurements, to analyze possible differences in behavior in subsets of differing weights, races, gestational ages, and postnatal ages and with or without phototherapy, in addition to assessing possible reliability limits, beyond which confirmation by invasive methods becomes necessary.

\section{Methods}

The study enrolled 200 newborn infants from the neonatal unit and maternity ward at the Centro de Atenção Integral à Saúde da Mulher (CAISM) and the Hospital Estadual de Sumaré (HES), both public tertiary institutions linked to the Universidade Estadual de Campinas (UNICAMP). Transcutaneous and plasma assays were performed just once for each patient, with a maximum interval of 30 min between them. A single $B C$ instrument was used, on the frontal region, and children on phototherapy had an area of approximately $5 \mathrm{~cm}^{2}$ shielded from the light with glued aluminum foil. After blood samples had been taken, capillary tubes were protected from ambient light, wrapped in aluminum and, after being centrifuged, were assayed for total bilirubin by direct spectrophotometry using a Unistat bilirubinometer (Leica) (BUL), model 10.310 C series 386-6.

Study variables were transcutaneous measurements (dependent) and total plasma bilirubin (independent), with co-variables investigated being birth weight measured on an electronic scale (less than or greater than 2,000 g), gestational age calculated by the Capurro or Ballard method (younger or older than 36 weeks), postnatal age (more or less than three complete days), skin color (white, black or mixed race) whether or not phototherapy was being used at the time of assay.

The sample size of 200 concomitant assays was calculated taking account of study design (validation of diagnostic test) with an estimated population sensitivity of $85 \%$ for the BC unit, ${ }^{4}$ with a desired absolute difference between sample and population sensitivities of $7 \%$ and with a type 1 error (a) of 0.05 . Statistical analysis was performed using SAS (version 8.02) and SPSS (version 10.0.7). Initially Pearson's correlation coefficient was calculated and, in order to assess agreement, a Bland-Altman graph was constructed. The influence of co-variables was analyzed using ANCOVA for repeated measures and then a series of receiver operating characteristic (ROC) curves were constructed, using the BUL readings as the reference standard, in order to identify a cutoff point beyond which $\mathrm{BC}$ readings are no longer trustworthy.
This study was approved by the Medical Ethics Committee at FCM/UNICAMP, and express consent was obtained from one of the parents of all participants.

\section{Results}

The majority of the neonates were white (66.5\%), $23.5 \%$ were mixed race and $10 \%$ black. At the time of bilirubin assay, $37.5 \%$ were on phototherapy and $62.5 \%$ were not. Mean gestational age was $35.7( \pm 3.73)$ weeks, birth weight was $2,330( \pm 930) \mathrm{g}$ and postnatal age was $3.25( \pm 1.73)$ days. Mean total plasma bilirubin was $8.10( \pm 4.17) \mathrm{mg} / \mathrm{dL}$ and transcutaneous bilirubin was $8.82( \pm 3.88) \mathrm{mg} / \mathrm{dL}$. The linear correlation coefficient was $0.92(p=0.0001)$, mean difference between the two assays was $0.72 \mathrm{mg} / \mathrm{dL}( \pm 1.57)$, with a $95 \%$ confidence interval varying from -2.42 to +3.86 (Figure 1).

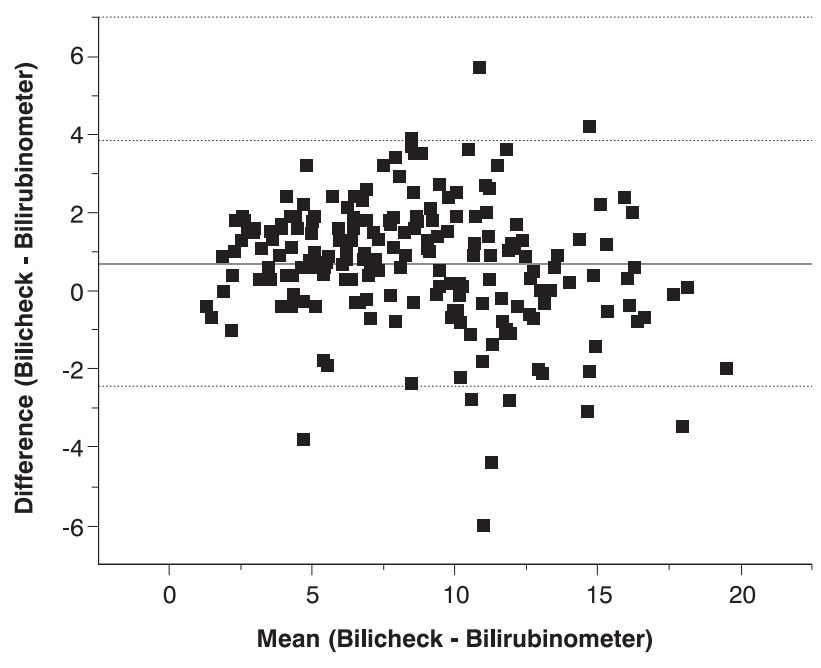

Figure 1 - Bland-Altman graph plotted from the differences between bilirubinometer assay and transcutaneous measurement with Bilicheck (mean difference of 0.72 $\mathrm{mg} / \mathrm{dL}$ and $95 \%$ confidence interval from -2.42 to $+3.86 \mathrm{mg} / \mathrm{dL}$ )

Table 1 contains comparisons of the two bilirubin measurement methods, broken down according to the variables studied. Taking the BUL as reference standard, a series of ROC curves were also constructed, revealing that the best cutoff point was at $14 \mathrm{mg} / \mathrm{dL}$. At this point the BC exhibited sensitivity of $88.2 \%$, specificity of $97.8 \%$, positive predictive value of $78.9 \%$ and negative predictive value of $98.9 \%$ and the area under the curve was 0.982 , with a $95 \%$ confidence interval from 0.963 to 1.002 .

\section{Discussion}

In our study we did not observe any significant interference by gestational age, birth weight, race or phototherapy with transcutaneous bilirubin measurements; while pointing out that the place where measurements were taken was conveniently protected from the action of light. The 
only variable that exhibited a small statistical difference ( $p=0.0030$ ) was postnatal age of less than 3 days, with the transcutaneous measurements being higher than the blood-based ones. This finding has also been reported by Ebbsen et al., ${ }^{7}$ and may be the result of an increase in bilirubin-albumin bonding and of cutaneous maturity as the days pass. In a study undertaken by Rubaltelli et al., ${ }^{4}$ neither postnatal nor gestational age had an influence of transcutaneous measurements and neither did race or birth weight. In that study BC was compared with high performance liquid chromatography (HPLC), considered the gold standard for bilirubin assays, but which, due to technical difficulties and elevated costs, is inappropriate for clinical use.

There are many variables involved in transcutaneous bilirubin measurement that preclude comparing the results of different studies. For example, the location where transcutaneous measurements are taken (frontal, sternal, etc.), the type of transcutaneous apparatus and the laboratory assay method used at different health services. To date, the only comparative study using BUL compared it with the Minolta JM-103. ${ }^{8}$ Comparison of our results with others in the literature that have used other bilirubin assay techniques is, therefore, problematic. The efficacy of the BUL for assaying total bilirubin in newborns has been proven for a long time and even today it is on a par with the best methods used in laboratories. ${ }^{9}$

Bilicheck ${ }^{\circledR}$ is an extremely advantageous alternative to traditional invasive assay methods, since it is painless and free from risk of infections secondary to blood sample collection. Furthermore, the results are available in just a few seconds, guaranteeing greater agility when caring for newborns. In our study, BC results overestimated bilirubin levels when compared with the BUL, making the risk of not

Table 1 - Comparisons of the two bilirubin measurement methods, broken down by the study variables

\begin{tabular}{|c|c|c|c|c|}
\hline Variables & $\mathbf{n}$ & Bilicheck (mg/dL) & Bilirubinometer $(\mathrm{mg} / \mathrm{dL})$ & p* \\
\hline Assays & 200 & $8.82 \pm 3.88$ & $8.10 \pm 4.17$ & 0.0802 \\
\hline Birth weight & & & & 0.6973 \\
\hline$<2,000 \mathrm{~g}$ & 85 & $6.03 \pm 2.55$ & $4.97 \pm 2.23$ & \\
\hline$\geq 2,000 \mathrm{~g}$ & 115 & $10.88 \pm 3.38$ & $10.41 \pm 3.75$ & \\
\hline Phototherapy & & & & 0.0597 \\
\hline Yes & 75 & $6.65 \pm 3.05$ & $5.62 \pm 2.73$ & \\
\hline No & 125 & $10.12 \pm 3.75$ & $9.59 \pm 4.19$ & \\
\hline Skin color & & & & 0.1560 \\
\hline White & 133 & $8.82 \pm 3.70$ & $7.97 \pm 3.94$ & \\
\hline Black & 20 & $8.32 \pm 3.53$ & $8.16 \pm 4.42$ & \\
\hline Mixed race & 47 & $9.03 \pm 4.54$ & $8.45 \pm 4.76$ & \\
\hline Postnatal age & & & & 0.0030 \\
\hline$<3$ days of life & 80 & $9.15 \pm 3.38$ & $8.23 \pm 3.56$ & \\
\hline$\geq 3$ days of life & 120 & $8.60 \pm 4.18$ & $8.02 \pm 4.55$ & \\
\hline Gestational age & & & & 0.4577 \\
\hline$<36$ weeks & 89 & $6.18 \pm 2.77$ & $5.16 \pm 2.50$ & \\
\hline$\geq 36$ weeks & 111 & $10.93 \pm 3.31$ & $10.46 \pm 3.72$ & \\
\hline
\end{tabular}

* ANCOVA for repeated measures. 
diagnosing important hyperbilirubinemia even smaller. Although there are other authors who have observed this finding, ${ }^{10}$ there are also studies in which the $B C$ underestimated serum bilirubin. ${ }^{7,11}$ This may be the result of interference from the laboratory method chosen and reinforces the need for a prior study of the most appropriate cutoff point for using the transcutaneous method.

With relation to the statistical analysis employed to compare the different methods; if the identity of the results suffers only minor variations, then it is possible to substitute one method for another. The accuracy of one method is best determined by concordance, but in clinical practice the maximum difference between assays must also be considered. We found differences of up to $3.8 \mathrm{mg} / \mathrm{dL}$, and perhaps the use of the mean of two consecutive transcutaneous measurements could reduce variation, without resulting in any great increase in costs, time or discomfort.

The fact that the results that best correlated were concentrated at lower values, led us to postulate that a cutoff point could be determined, below which the $B C$ could be safely used. A series of ROC curves were plotted in search of this point, and it was found that $14 \mathrm{mg} / \mathrm{dL}$ exhibited the best combination of sensitivity and specificity. Above this level it becomes necessary to confirm readings with the BUL in order to ensure taking the correct therapeutic measures with safety. This cutoff point is lower than has been suggested by some authors; ${ }^{5,6}$ although, in the opinion of others, ${ }^{12}$ BC could entirely replace blood-based assays, irrespective of bilirubinemia levels.

In clinical practice, the majority of bilirubin assay results are below $14 \mathrm{mg} / \mathrm{dL}$. Employing BC resulted in up to 55\% less blood tests in a study published by Samanta et al. ${ }^{11}$ The use of this equipment for monitoring, although offering advantages, brings up the inconvenience of increased cost when compared with assays performed with the BUL. Nevertheless, a recent study demonstrated safe reutilization of the BC's calibration capsules, which, if reused rather than changed for every assay, significantly reduces the assay cost, making it more accessible in clinical practice. ${ }^{13}$

Further studies should be carried out that compare, not only the technical performance of transcutaneous instruments, but also assess their cost/benefit relationship.

\section{Acknowledgements}

We would like to thank the Statistics Department of the Research Commission at the UNICAMP Medical Sciences Faculty for their invaluable analysis of our database.

\section{References}

1. Hannemann RE, Schreiner RL, DeWitt DP, Norris SA, Glick MR. Evaluation of the Minolta bilirubin meter as a screening device in white and black infants. Pediatrics. 1982;69:107-9.

2. Bertini G, Rubaltelli FF. Non-invasive bilirubinometry in neonatal jaundice. Semin Neonatol. 2002;7:129-33.

3. Bhutani VK, Gourley GR, Adler S, Kreamer B, Dalin C, Johnson $\mathrm{LH}$. Noninvasive measurement of total serum bilirubin in a multiracial predischarge newborn population to assess the risk of severe hyperbilirubinemia. Pediatrics. 2000;106:E17.

4. Rubaltelli FF, Gourley GR, Loskamp N, Modi N, Roth-Kleiner M, Sender A, et al. Transcutaneous bilirubin measurement: a multicenter evaluation of a new device. Pediatrics. 2001; 107:1264-71.

5. Engle WD, Jackson GL, Sendelbach D, Manning D, Frawley WH. Assessment of transcutaneous device in the evaluation of neonatal hyperbilirubinemia in a primarily Hispanic population. Pediatrics. 2002;110:61-7.

6. Szabo P, Wolf M, Bucher HV, Haensse D, Fauchere JC, Arlettaz R. Assessment of jaundice in preterm neonates: comparison between clinical assessment, two transcutaneous bilirubinometers and serum bilirubin values. Acta Paediatr. 2004; 93:1491-5.

7. Ebbesen F, Rasmussen LM, Wimberley PD. A new transcutaneous bilirubinometer, Bilicheck, used in the neonatal intensive care unit and the maternity ward. Acta Paediatr. 2002;91:203-11.

8. Sanpavat S, Nuchprayoon I. Noninvasive transcutaneous bilirubin as a screening test to identify the need for serum bilirubin assessment. J Med Assoc Thai. 2004;87:193-8.

9. Williams RA, Pits LL 2nd, Weinerth JL, Dimmette RM. Clinical laboratory evaluation of the American optical Bilirubinometer. J Pediatr. 1971;79:671-4.

10. Sanpavat S, Nuchprayoon I. Comparison of two transcutaneous bilirubinometers- Minolta AirShields jaundice meter JM103 and Spectrx Bilicheck- in Thai neonates. Southeast Asian J Trop Med Public Health. 2005;36:1533-7.

11. Samanta S, Tan M, Kissack C, Nayak S, Chittick R, Yoxall C. The value of Bilicheck as a screening tool for neonatal jaundiced in term and near-term babies. Acta Paediatr. 2004;93:1486-90.

12. Janjindamai W, Tansantiwong T. Accuracy of transcutaneous bilirubinometer estimates using Bilicheck in Thai neonates. J Med Assoc Thai. 2005;88:187-90.

13. Facchini FP. Avaliação da variabilidade da dosagem transcutânea de bilirrubina pelo Bilicheck em neonatos. Rev Paul Pediatria. 2006;24:149-54.

Correspondence:

Maria das Graças da Cunha Leite

Rua Ciro Lagazzi, 1102, Parque Santa Cândida

CEP 13603-027 - Araras, SP - Brazil

Tel.: +55 (19) 3544.6634, +55 (19) 8129.7031

Fax: +55 (19) 3883.8926

E-mail: mgcleite@gmail.com 